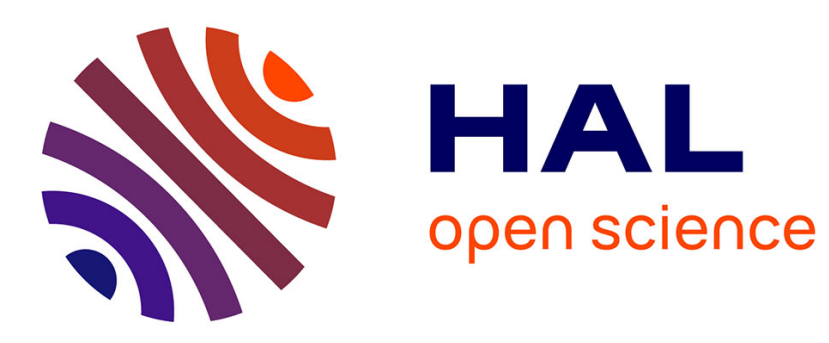

\title{
Planning and Nonlinear Adaptive Control for an Automated Overtaking Maneuver
}

\author{
Plamen Petrov, Fawzi Nashashibi
}

\section{To cite this version:}

Plamen Petrov, Fawzi Nashashibi. Planning and Nonlinear Adaptive Control for an Automated Overtaking Maneuver. ITSC 2011 - 14th International IEEE Conference on Intelligent Transportation Systems, The George Washington University, Oct 2011, Washington D.C., United States. pp.662-667, 10.1109/ITSC.2011.6083025 . hal-00663044

\section{HAL Id: hal-00663044 \\ https://hal.inria.fr/hal-00663044}

Submitted on 26 Jan 2012

HAL is a multi-disciplinary open access archive for the deposit and dissemination of scientific research documents, whether they are published or not. The documents may come from teaching and research institutions in France or abroad, or from public or private research centers.
L'archive ouverte pluridisciplinaire $\mathbf{H A L}$, est destinée au dépôt et à la diffusion de documents scientifiques de niveau recherche, publiés ou non, émanant des établissements d'enseignement et de recherche français ou étrangers, des laboratoires publics ou privés. 


\title{
Planning and Nonlinear Adaptive Control for an Automated Overtaking Maneuver
}

\author{
Plamen Petrov and Fawzi Nashashibi
}

\begin{abstract}
In this paper, we present a nonlinear adaptive controller for a two-vehicle automated overtaking maneuver. We consider the problem of a three-phase overtaking without the use of information obtained from road infrastructure. The only information available for feedback control is the current relative inter-vehicle position and orientation. Reference trajectories for every phase are generated in real time. An update control law for the automated overtaking vehicle is designed that allows tracking desired trajectories in the presence of unknown velocity of the overtaken vehicle. Simulation results are included to illustrate the performance of the proposed controller.
\end{abstract}

\section{INTRODUCTION}

$\mathrm{T}$ HIS paper presents a nonlinear adaptive controller for a two-vehicle automated overtaking maneuver. Various control laws for different maneuvers of automated vehicles has been designed and analyzed during the last two decades. The ultimate goal in automating the driving process is to reduce accidents caused by human error and increase safety. While considerable research work has been reported on lane keeping [1] and lane change maneuver [2,3], the problem of automated overtaking has attracted less attention. In contrast to lane keeping and lane change maneuvers, the overtaking is a composition of three consecutive maneuvers: lane change, followed by trajectory tracking and again a lane change, which have to be planned and coordinated. A twolayer controller architecture for overtaking maneuver is presented in [4]. The lower level consists of two fuzzy steering controllers for path tracking and lane change, and the high-level is to evaluate the necessity and possibility of overtaking, and to switch between the low-level controllers. An on-road demonstration of cooperative driving solutions and, in particular, overtaking maneuver by autonomous road vehicles designed for the cities is reported in [5]. In [6], two methods for real-time trajectory planning applied to automated driving, which are based on partial motion planning approach and $5^{\text {th }}$ order polynomial interpolation method, respectively are presented. They have been implemented for the HAVEit European project. A smooth optimal lane-change trajectory for the overtaking maneuver is presented in [7].

The authors acknowledge the financial support from the FP7 EU project HAVEit.

P. Petrov is with the Faculty of Mechanical Engineering, Technical University of Sofia, 1000 Sofia, Bulgaria, (phone: 3592-965-3271; e-mail: ppetrov@ tu-sofia.bg).

F. Nashashibi is with the Institut National de la Recherche en Informatique et Automatique (INRIA), 78153 Rocquencourt, France, (email: Fawzi.Nashashibi@inria.fr)
In this paper, we consider the problem of automated overtaking without the use of road infrastructure. An important issue in designing feedback controllers for overtaking maneuvers is to decide the desired trajectory of the overtaking vehicle. The approach used in this paper, is inspired by our previous works $[8,9]$, in the sense that it consists in consecutive tracking reference virtual points, which are positioned at desired a priory known distances from the overtaken vehicle with a virtual reference point attached to the overtaking vehicle. In addition, similar to [6], reference trajectories for the desired motion of the overtaking vehicle during the overtaking maneuver are generated using polynomial functions. We consider an automated overtaking maneuver with only the current intervehicle position and orientation available for feedback control. An adaptive nonlinear controller for the overtaking vehicle is designed that allows tracking desired trajectories in the presence of unknown velocity of the overtaken vehicle.

The organization of the paper is as follows: In Section II, the mathematical description of a three-phase overtaking maneuver suitable for feedback control is derived. The Problem formulation is given in Section III. In Section IV, a nonlinear adaptive control law is designed. Section V contains simulation results. Conclusions are presented in Section VI.

\section{MATHEMATIC MODEL}

\section{A. Three-Phase Overtaking Maneuver}

In this paper, the overtaking involving two vehicles is established as a three-phase maneuver (illustrated in Fig. 1).

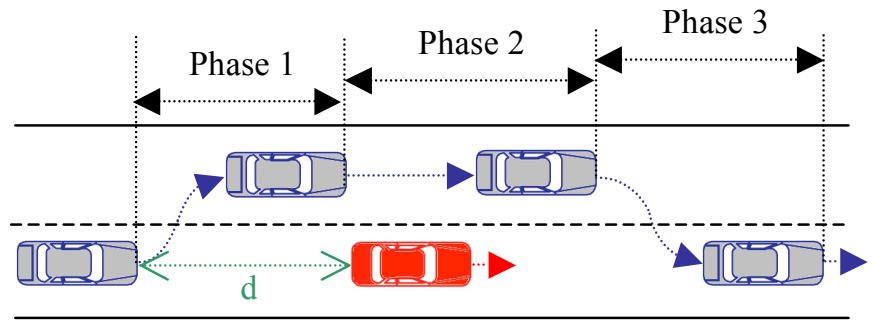

Fig. 1. A three-phase overtaking maneuver involving two vehicles

We assume that the following conditions are met:

--The overtaken vehicle is moving along a rectilinear route with a constant velocity.

--The left lane is free and there is enough space to complete the overtaking maneuver. 
--The linear velocity of the overtaken vehicle is unknown.

--The only information available for feedback control is the relative inter-vehicle position and orientation.

A brief description of the three-phase overtaking scenario considered in this paper is provided below. Assume that the automated vehicle has to pass the preceding car at distance $d$ ahead. The first phase consists of "lane-change"-type maneuver. Starting with some initial conditions, the overtaking vehicle diverts from the lane and tracks a given reference trajectory for a given time period and has to reach a preselected position behind the overtaken vehicle, (Fig. 1). At the end of the first time period, the guidance program switches automatically from Phase 1 to Phase 2. The second phase consists of driving straight in the adjacent lane along the overtaken vehicle at a prescribed lateral distance. The vehicle tracks again a given reference trajectory for a given time period until the overtaken vehicle has been passed and the overtaking vehicle reaches a preselected position on the left side of the overtaken vehicle, (Fig. 1). During the third phase, given a reference trajectory for the third phase, the overtaking vehicle returns to the lane and has to reach a preselected position in front of the overtaken vehicle. The desired position and linear velocity of the overtaking vehicle with respect to the overtaken vehicle at the end of every phase, as well the phase duration, are determined to satisfy the operational requirements imposed on such a maneuver. A reference trajectory for the overtaking vehicle is generated in real time for every phase. At the beginning of every phase, the desired initial position and velocity of the overtaking vehicle coincides with its current position and velocity. In such way, there is no restriction for the initial position, orientation, linear and angular velocities of the overtaking vehicle in the beginning of every phase of the overtaking maneuver. However, from a practical point of view, a minimal inter-vehicle distance $d$ is introduced for the beginning of the maneuver, in order to meet some geometrical and dynamical limitations of the vehicles, (for example, limitations for the front-wheel steering angle).

\section{B. Relative Kinematics}

A schematic plan view of an overtaking maneuver involving two vehicles is shown in Fig. 1. We assume that the vehicles have non-deformable wheels which roll on a horizontal plane without slipping. In order to describe the position and orientation of the vehicles during the overtaking maneuver, we assign the following coordinate frames, (Fig. 2):

- Fxy - inertial coordinate frame in the plane of motion;

- $\quad A_{i} x_{A i} y_{A i},(i=1,2)$ - vehicle coordinate frames located at the center of the rear vehicle axels of the overtaken and the overtaking vehicle, respectively, where $x_{i}$ is along the longitudinal base of the vehicle;

- $\quad R_{i} x_{R i} y_{R i},(i=1,2,3)$ - virtual reference frames rigidly attached to the overtaken vehicle and located at specific places, as shown in Fig. 2. The coordinates of the origins $R_{i}$ with respect to $A_{1} x_{A l} y_{A l}$ are $\left(L_{l t}^{i} L_{l n}^{i}\right),(i$
$=1,2,3)$ and the corresponding axes are parallel to those of $A_{1} x_{A l} y_{A l}$.

- $L x_{L} y_{L}$ - coordinate frame rigidly linked to the overtaking vehicle and located at the mid-point of the front bumper of the vehicle at a distance $L_{2}$ from point $A_{2}$, where the $x_{L}$ axis is in direction of the longitudinal vehicle axle.

For brevity, in the exposition which follows, we will derive in details the relative inter-vehicle kinematics with respect to the first phase of the overtaking maneuver (lane change maneuver). Similar relationships can be also derived for Phase 2 and Phase 3.

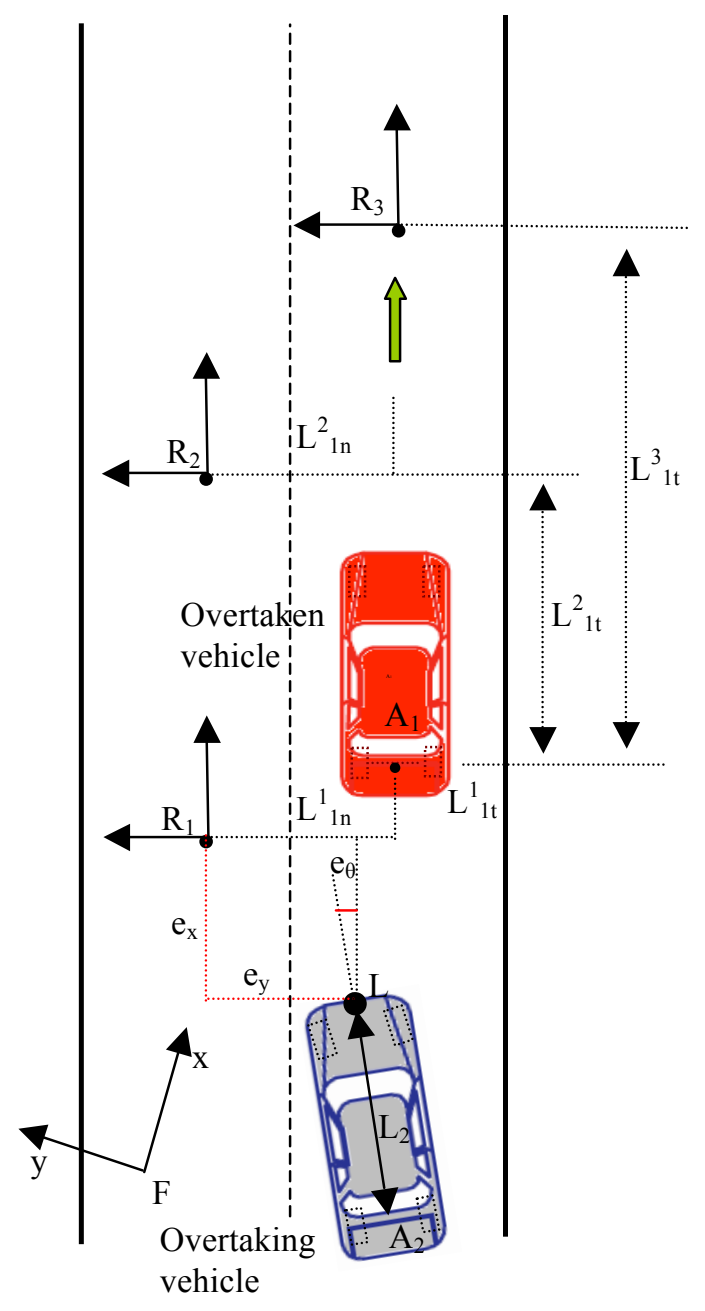

Fig. 2. Coordinate frame assignment

\section{1) Phase 1:}

Let us denote by $p_{A 1}=\left[\begin{array}{ll}x_{A 1} & y_{A 1}\end{array}\right]^{T} \in \mathfrak{\Re}^{2}$ the coordinates of point $A_{l}$ with respect to the frame $F x y$; the coordinates of point $R_{I}$ with respect to the frame $F x y$ by $p_{R 1}=\left[\begin{array}{ll}x_{R 1} & y_{R 1}\end{array}\right]^{T} \in \Re^{2}$, and the orientation of $A_{1} x_{1} y_{l}$ with respect to $F x y$ by $\theta_{R}$. If the vector $d_{A 1}^{R 1}=\left[\begin{array}{ll}-L_{1 t}^{1} & L_{1 n}^{1}\end{array}\right] \in \mathfrak{R}^{2}$ is the vector from the origin $A_{l}$ to the origin $R_{l}$ expressed in the coordinate frame $A_{1} x_{I} y_{1}$, then the coordinates of points $R_{I}$ 
and $A_{l}$ are related by

$$
p_{R 1}=p_{A 1}+R_{r} d_{A 1}^{R 1}
$$

where $R_{r} \in S O(2)$ is orthogonal matrix.

Similarly, we denote by $p_{A 2}=\left[\begin{array}{ll}x_{A 2} & y_{A 2}\end{array}\right]^{T} \in \mathfrak{R}^{2}$ the coordinates of point $A_{2}$ with respect to the frame Fxy; the coordinates of point $L$ with respect to the frame Fxy by $p_{L}=\left[\begin{array}{ll}x_{L} & y_{L}\end{array}\right]^{T} \in \Re^{2}$, and the orientation of $A_{2} x_{2} y_{2}$ with respect to $F x y$ by $\theta$. If the vector $d_{A 2}^{L}=\left[\begin{array}{ll}L_{2} & 0\end{array}\right]^{T} \in \Re^{2}$ is the vector from the origin $A_{2}$ to the origin $L$ expressed in the coordinate frame $A_{2} x_{A 2} y_{A 2}$, then the coordinate of points $L$ and $A_{2}$ are related by

$$
p_{L}=p_{A 2}+R d_{A 2}^{L}
$$

where $R \in S O(2)$ is orthogonal matrix.

The coordinate frames $R_{l} x_{R I} y_{R I}$ and $L x_{L} y_{L}$ are defined to describe the relative inter-vehicle kinematics during the first phase of the overtaking maneuver. For this purpose, the coordinates and orientation of the frame $L x_{L} y_{L}$ in the coordinate frame $R_{I} x_{R I} y_{R I}$ can be expressed as follows

$$
\left[\begin{array}{l}
e_{x} \\
e_{y} \\
e_{\theta}
\end{array}\right]=\left[\begin{array}{ccc}
\cos \theta_{R} & \sin \theta_{R} & 0 \\
-\sin \theta_{R} & \cos \theta_{R} & 0 \\
0 & 0 & 1
\end{array}\right]\left[\begin{array}{c}
x_{L}-x_{R 1} \\
y_{L}-y_{R 1} \\
\theta-\theta_{R}
\end{array}\right]
$$

where $e=\left[\begin{array}{lll}e_{x} & e_{y} & e_{\theta}\end{array}\right]^{T} \in \mathfrak{R}^{3}$ is the error posture.

Differentiating (3) with respect to time and taking into account equations (1) and (2), after some work, the intervehicle kinematics in error coordinates is obtained as follows

$$
\begin{aligned}
{\left[\begin{array}{l}
\dot{e}_{x} \\
\dot{e}_{y} \\
\dot{e}_{\theta}
\end{array}\right] } & =\left[\begin{array}{ccc}
\cos e_{\theta} & 0 & -L_{2} \sin e_{\theta} \\
\sin e_{\theta} & 0 & L_{2} \cos e_{\theta} \\
0 & 0 & 1
\end{array}\right]\left[\begin{array}{c}
v_{x} \\
0 \\
\omega
\end{array}\right]-\left[\begin{array}{ccc}
1 & 0 & -L_{1 n}^{1} \\
0 & 0 & -L_{1 t}^{1} \\
0 & 0 & 1
\end{array}\right]\left[\begin{array}{c}
v_{R x} \\
0 \\
\omega_{R}
\end{array}\right] \\
& +\omega_{R}\left[\begin{array}{ccc}
0 & 1 & 0 \\
-1 & 0 & 0 \\
0 & 0 & 0
\end{array}\right]\left[\begin{array}{l}
e_{x} \\
e_{y} \\
e_{\theta}
\end{array}\right]
\end{aligned}
$$

where $v_{x}$ and $v_{R x}$ are the longitudinal components of the linear velocities of points $A_{1}$ and $A_{2}$, respectively; $\omega:=\dot{\theta}$ and $\omega_{R}:=\dot{\theta}_{R}$ are the angular velocities of the overtaking and overtaken vehicle, respectively.

We note that based on the assumption of pure rolling without lateral sliding of the wheels, the nonholonomic constraints imply that in (4) the components of the velocities of points $A_{1}$ and $A_{2}$ in direction of the rear vehicle axles are equal to zero, i.e., $v_{y}=v_{R y}=0$.

Remark 1: The front wheel steering angle $\alpha$ of the overtaking vehicle which is a control input for the system (4) is related to the angular velocity of the vehicle as follows

$$
\alpha=a \tan \left(l_{2} \frac{\omega}{v_{x}}\right)
$$

In the following, we consider an overtaking maneuver during rectilinear motion of the overtaken vehicle. In this case, the angular velocity of the overtaking vehicle $\omega_{R}=0$.

Let us consider the following change of inputs in (4)

$$
\left[\begin{array}{l}
u_{1} \\
u_{2}
\end{array}\right]=\left[\begin{array}{cc}
\cos e_{\theta} & -L_{2} \sin e_{\theta} \\
\operatorname{sine}_{\theta} & \mathrm{L}_{2} \cos e_{\theta}
\end{array}\right]\left[\begin{array}{c}
v_{x} \\
\omega
\end{array}\right]
$$

where one easy verify that the transformation matrix is nonsingular when $L_{2} \neq 0$. Using (6), in case of rectilinear motion of the overtaken vehicle, the inter-vehicle kinematic equations (4) can be written in the form

$$
\begin{aligned}
& \dot{e}_{x}=u_{1}-v_{R x} \\
& \dot{e}_{y}=u_{2} \\
& \dot{e}_{\theta}=-\frac{\sin e_{\theta}}{L_{2}} u_{1}+\frac{\cos e_{\theta}}{L_{2}} u_{2}
\end{aligned}
$$

\section{2) Phase 2 and Phase 3:}

During the Phase 2, the vector $d_{A 1}^{R 2}$ which is the vector from the origin $A_{1}$ to the origin $R_{2}$ expressed in the coordinate frame $A_{1} x_{A 1} y_{A 1}$ is set to be $d_{A 1}^{R 2}=\left[\begin{array}{ll}L_{1 t}^{2} & L_{1 n}^{2}\end{array}\right]^{T} \in \Re^{2}$, where $L_{1 n}^{2}=L_{1 n}^{1}$. In the same way, for Phase 3, vector $d_{A 1}^{R 3}$ is set as $d_{A 1}^{R 3}=\left[\begin{array}{ll}L_{1 t}^{3} & 0\end{array}\right]^{T} \in \mathfrak{R}^{2}$, (Fig. 2).

\section{PROBLEM FORMULATION}

In this paper, we consider autonomous overtaking maneuver without any information obtained from road infrastructure or communicated from the overtaken vehicle. The only information that the overtaking (robot) vehicle can use for feedback control is the current relative position and orientation with respect to the overtaken vehicle given by (3) which are obtained from onboard sensors.

\section{A. Trajectory Planning}

We consider in details the problem of generating smooth trajectories for point to point motion in terms of the posture errors $\left(e_{x}, e_{y}\right)$ with respect to the moving reference frame $R_{I} x_{R I} y_{R I}$ which is rigidly linked to the overtaken vehicle. Assuming rectilinear motion at constant velocity of the overtaken vehicle, we are interested in overtaking scenario for the first phase, when the overtaking vehicle starts the maneuver from an arbitrary initial position and orientation behind the overtaken vehicle with given (arbitrary) velocity. In the end of the first phase, the two vehicles have to be parallel and in the same time, the desired position of the overtaking vehicle behind the overtaken vehicle has to be shifted at distances $L_{1 t}^{1}$ and $L_{1 n}^{1}$ in longitudinal and lateral direction, respectively, (Fig. 2). These distances are determined from considerations of safety driving and sensor requirements (in particular, $L_{1 t}^{1}$ may be equal to zero). In addition, the relative inter-vehicle velocity in longitudinal direction must be equal to a prescribed non-zero value. For this end, we suppose that at time $t_{0}$, the state variables satisfy

$$
\begin{array}{ll}
e_{x}^{d}\left(t_{0}\right)=e_{x 0} & e_{y}^{d}\left(t_{0}\right)=e_{y 0} . \\
\dot{e}_{x}^{d}\left(t_{0}\right)=\dot{e}_{x 0} & \dot{e}_{y}^{d}\left(t_{0}\right)=\dot{e}_{y 0}
\end{array}
$$


At time $t_{f}$, we wish to attain the values

$$
\begin{aligned}
& e_{x}^{d}\left(t_{f}\right)=0 \quad e_{y}^{d}\left(t_{f}\right)=0 \\
& \dot{e}_{x}^{d}\left(t_{f}\right)=\Delta v_{R x}\left(t_{f}\right), \quad \dot{e}_{y}^{d}\left(t_{f}\right)=0
\end{aligned}
$$

where $\Delta v_{R x}\left(t_{f}\right)$ is the prescribed relative non-zero intervehicle velocity in $x_{R}$-direction at $t_{f}$, i.e., the overtaking vehicle has to start the second phase of the overtaking maneuver at prescribed velocity which is higher compared to the velocity of the overtaken vehicle. The time $t_{f}$, is determined from considerations of vehicle's power and acceleration capabilities. The initial values of the program errors $\left(e_{x}^{d}\left(t_{0}\right), e_{y}^{d}\left(t_{0}\right)\right)$ are equal of the current values $\left(e_{x}\left(t_{0}\right), e_{y}\left(t_{0}\right)\right)$ at $t=t_{0}$. However, from a practical point of view, the following conditions have to be satisfied: $a_{x} \leq e_{x}\left(t_{0}\right) \leq b_{x}, a_{y} \leq e_{y}\left(t_{0}\right) \leq b_{y}$, where $a_{x}, b_{x}, a_{y}$, and $b_{y}$ are determined from considerations of safety driving, sensor requirements, and vehicle's power and acceleration capabilities. In this paper, we consider cubic desired trajectories of the form

$$
\begin{aligned}
& e_{x}^{d}(t)=a_{0 x}+a_{1 x}\left(t-t_{0}\right)+a_{2 x}\left(t-t_{0}\right)^{2}+a_{3 x}\left(t-t_{0}\right)^{3} \\
& e_{y}^{d}(t)=a_{0 y}+a_{1 y}\left(t-t_{0}\right)+a_{2 y}\left(t-t_{0}\right)^{2}+a_{3 y}\left(t-t_{0}\right)^{3}
\end{aligned}
$$

Differentiating (10) with respect to time, we obtain quadratic polynomials with respect to the derivatives $\dot{e}_{x}^{d}(t)$ and $\dot{e}_{y}^{d}(t)$ :

$$
\begin{aligned}
& \dot{e}_{x}^{d}(t)=a_{1 x}+2 a_{2 x}\left(t-t_{0}\right)+3 a_{3 x}\left(t-t_{0}\right)^{2} . \\
& \dot{e}_{y}^{d}(t)=a_{1 y}+2 a_{2 y}\left(t-t_{0}\right)+3 a_{3 y}\left(t-t_{0}\right)^{2}
\end{aligned} .
$$

Using (8), (9), (10) and (11), we obtain 8 equations for the eight unknown coefficients $a_{i x}$ and $a_{i y},(i=1,2,3,4)$. Similar expressions for the desired trajectories can be assigned for Phase 2 and 3.

\section{B. Problem Statement}

We assume that we are able to measure the posture coordinates $e=\left[\begin{array}{lll}e_{x} & e_{y} & e_{\theta}\end{array}\right]^{T} \in \mathfrak{R}^{3}$ defined by (3), but the linear velocity $v_{R x}$ of the overtaken vehicle is unknown constant parameter.

Given the inter-vehicle kinematics in error coordinates (7) the control objective for Phase 1 is to asymptotically regulate to zero the coordinates of reference point $L$ of the overtaking vehicle with respect to the coordinate frame $R_{I}$ attached to the overtaken vehicle according to desired trajectories (10) for the error coordinates $\left(e_{x}, e_{y}\right)$ with initial and final conditions given by (8)-(9). Similar objectives can be formulated for Phase 2 and 3 where the virtual reference points associated with the overtaken vehicle are $R_{2}$ and $R_{3}$, respectively.

\section{AdAPtive CONTROL Design}

In this paper, we consider the problem of controlling the motion of the overtaking vehicle during the overtaking maneuver, where the overtaken vehicle moves straight with constant velocity.
Consider again in details the Phase 1 of the overtaking maneuver. We make the following change of coordinates

$$
\begin{aligned}
& x_{e}=e_{x}-e_{x}^{d} \\
& y_{e}=e_{y}-e_{y}^{d}
\end{aligned}
$$

where the posture error coordinates $\left(e_{x}, e_{y}\right)$ are defined by (3), and the desired trajectories $\left(e_{x}^{d}(t), e_{y}^{d}(t)\right)$ are given by (10).

The system (7) in the new coordinates becomes

$$
\begin{aligned}
& \dot{x}_{e}=u_{1}-v_{R x}-\dot{e}_{x}^{d} \\
& \dot{y}_{e}=u_{2}-\dot{e}_{y}^{d} \\
& \dot{e}_{\theta}=-\frac{\sin e_{\theta}}{L_{2}} u_{1}+\frac{\cos e_{\theta}}{L_{2}} u_{2}
\end{aligned}
$$

The adaptive control design is based on a reduced-order system composed of the first two equations of (13) rewritten below for clarity of exposition

$$
\begin{aligned}
& \dot{x}_{e}=u_{1}-v_{R x}-\dot{e}_{x}^{d} . \\
& \dot{y}_{e}=u_{2}-\dot{e}_{y}^{d}
\end{aligned}
$$

Consider the system (14) and assume that the velocity of the overtaken vehicle $v_{R x}=c t e>0$ is unknown constant parameter. The control problem consists in finding an adaptive feedback control law for the system (14) with inputs $\left(u_{1}, u_{2}\right)$ such that

$$
\lim _{t \rightarrow \infty}\left(x_{e}(t)\right)=0 \text { and } \lim _{t \rightarrow \infty}\left(x_{e}(t)\right)=0 .
$$

Consider the control

$$
\begin{aligned}
& u_{1}=\hat{v}_{R x}+\dot{e}_{x}^{d}-k_{x} x_{e} \\
& u_{2}=\dot{e}_{y}^{d}-k_{y} y_{e}
\end{aligned}
$$

where $k_{x}$ and $k_{y}$ are positive gains. We chose the following Lyapunov function candidate

$$
V=\frac{1}{2} x_{e}^{2}+\frac{1}{2} y_{e}^{2}+\frac{1}{2 \gamma_{v}} \widetilde{v}_{R x}^{2}
$$

where

$$
\widetilde{v}_{R x}=\widetilde{v}_{R x}-v_{R x}
$$

is the parameter error and $\gamma_{v}=c t e>0$ is the adaptation gain. Using (14), (16) and (18), the derivative of $V$ is obtained in the form

$$
\dot{V}=-k_{x} \dot{x}_{e}^{2}-k_{y} \dot{y}_{e}^{2}+\widetilde{v}_{R x}\left(x_{e}+\frac{1}{\gamma_{v}} \dot{\hat{v}}_{R x}\right) .
$$

Choosing the update law as

$$
\dot{\hat{v}}_{R x}=-\gamma_{v} x_{e}
$$

we get for the derivative of $V$

$$
\dot{V}=-k_{x} \dot{x}_{e}^{2}-k_{y} \dot{y}_{e}^{2} \leq 0 .
$$

The resulting closed-loop adaptive system becomes

$$
\begin{aligned}
& \dot{x}_{e}=-k_{x} x_{e}+\widetilde{v}_{R x} \\
& \dot{y}_{e}=-k_{y} y_{e} \\
& \dot{\widetilde{v}}_{R x}=-\gamma_{v} x_{e}
\end{aligned} .
$$

Proposition 1: Assume that the linear velocity of the overtaken vehicle is bounded unknown constant parameter 
$v_{R x}=$ cte $>0$ and $L_{2} \neq 0$. If the control law given by (16) is applied to (14), where the velocity estimate $\hat{v}_{R x}$ is obtained from the parameter update law (20), the origin $z=\left[x_{e}, y_{e}, \widetilde{v}_{R x}\right]^{T}=0$ of the closed-loop system (22) is asymptotically stable.

Proof. The system (22) has an equilibrium point at the origin. The function (17) is continuously differentiable and positive definite and its derivative (21) along the trajectories of the system is negative semi-definite. From (21), it follows that (17) is non-increasing, $(V(t) \leq V(0))$, and this in turn implies that $x_{e}(t), y_{e}(t)$ and $\widetilde{v}_{R x}(t)$ are uniformly bounded with respect to the initial conditions.

To characterize the set $S=\left\{z \in \mathfrak{R}^{3} \mid \dot{V}(z)=0\right\}$, note that

$$
\dot{V}(z)=0 \Rightarrow x_{e}=0 \text { and } y_{e}=0 .
$$

Hence $S=\left\{z \in \mathfrak{R}^{3} \mid x_{e}=0 ; \quad y_{e}=0\right\} . \quad$ To prove asymptotic stability of the equilibrium point $\mathrm{z}=0$, we use the LaSalle invariance principle [10]. Suppose that $z(t)$ is a trajectory that belongs identically to $S$. From (22), we have

$$
\begin{aligned}
& x_{e}(t) \equiv 0 \Rightarrow \dot{x}_{e} \equiv 0 \Rightarrow \widetilde{v}_{R x} \equiv 0 \\
& y_{e}(t) \equiv 0 \Rightarrow \dot{y}_{e} \equiv 0 \\
& x_{e}(t) \equiv 0 \Rightarrow \dot{\widetilde{v}}_{R x} \equiv 0
\end{aligned}
$$

Therefore, the only solution that can stay in $S$ is the trivial solution $z(t)=0$ and the origin is asymptotically stable

Since the dynamics of $e_{\theta}$ was not taken into account in the feedback control design, the next step in the stability analysis is to establish that $e_{\theta}$ is bounded in the interval $\left[t_{0}\right.$, $\left.t_{f}\right]$. From (13), using the control (16), the third equation for $e_{\theta}$ takes the form of a perturbed system

$$
\dot{e}_{\theta}=f\left(e_{\theta}\right)+g\left(t, e_{\theta}\right)
$$

where the nominal system is given by

$$
\dot{e}_{\theta}=f\left(e_{\theta}\right)=-\frac{v_{R x}}{L_{2}} \sin e_{\theta},
$$

and the perturbation term is obtained in the form

$$
\begin{aligned}
g\left(t, e_{\theta}\right)= & \frac{1}{L_{2}}\left[-\left(\widetilde{v}_{R x}-k_{x} x_{e}\right) \sin e_{\theta}-k_{y} y_{e} \cos e_{\theta} .\right. \\
& \left.-\dot{e}_{x}^{d} \sin e_{\theta}+\dot{e}_{y}^{d} \cos e_{\theta}\right]
\end{aligned}
$$

Since $x_{e}(t), y_{e}(t)$ and $\widetilde{v}_{R x}(t)$ are uniformly bounded, $\sin \left(e_{\theta}\right)$ and $\cos \left(e_{\theta}\right)$ are bounded functions, and the polynomial functions (11) are bounded within the interval $\left[t_{0}, t_{f}\right]$, it follows that

$$
\left\|g\left(t, e_{\theta}\right)\right\| \leq \lambda
$$

in the domain of interest, ( $\lambda$ is a positive constant).

The point $\mathrm{e}_{\theta}=0$ is an exponentially stable equilibrium point for the nominal system (26), which can be easy proved by using, for example, the Lyapunov function $W=1-\cos e_{\theta}$. Therefore, $e_{\theta}$ is bounded and $e_{\theta}(t) \rightarrow 0$ as $t \rightarrow \infty$. If $e_{\theta n}(t)$ and $e_{\theta p}(t)$ are the solutions of the nominal and perturbed systems (26) and (25), respectively, using the Gronwall-Bellman inequality [10], we have on the compact time interval $\left[t_{0}, t_{f}\right]$

$$
\begin{aligned}
\left\|e_{\theta n}(t)-e_{\theta_{p}}(t)\right\| \leq & \left\|e_{\theta n}\left(t_{0}\right)-e_{\theta 0}(t)\right\| \exp \left[l\left(t-t_{0}\right)\right] \\
& +\frac{\lambda}{l}\left\{\exp \left[l\left(t-t_{0}\right)\right]-1\right\}
\end{aligned}
$$

where $l$ is a Lipschitz constant for the nominal system (26). Hence, $e_{\theta p}(t)$ is bounded on the time interval $\left[t_{0}, t_{f}\right]$.

As mentioned above, at the end of the first time period, the guidance program switches automatically from Phase 1 to Phase 2. The same approach can be used to derive feedback controllers for the overtaking vehicle for Phase 2 and Phase 3.

\section{Simulation Results}

To illustrate the effectiveness of the proposed controller, several simulations are carried out in order to evaluate the inter-vehicle behavior and tracking accuracy during the three-phase overtaking maneuver. In the simulation using MATLAB, a planar bicycle 2DOFs vehicle model is used. The speed of the overtaken vehicle during the three phases of the maneuver is constant and set to be $v_{A}=4 \mathrm{~m} / \mathrm{s}$. The duration of every phase of the maneuver is chosen to be $t_{\text {in }}$ $=5 \mathrm{~s}$. The coordinates of the reference frames $R_{i} x_{R i} y_{R i},(i=$ $1,2,3)$ with respect to the coordinate frame $A_{1} x_{A l} y_{A 1}$ attached to the mid-point of the rear vehicle axle of the overtaken vehicle for every phase of the three-phase overtaking maneuver is provided in Table I.

The longitudinal vehicle base of the overtaking vehicle was chosen to be $L_{2}=2 m$. The initial position and orientation of the overtaking vehicle in the inertial frame Fxy are $x_{A 2}(0)=0 ; y_{A 2}(0)=0 ; \theta(0)=0$. The initial position and orientation of the overtaken vehicle in Fxy are $x_{A I}(8)=$ $0 ; y_{A I}(0)=0 ; \theta_{R}(0)=0$. The desired inter-vehicle distance in the beginning of the maneuver is set to be $6 \mathrm{~m}$.

In the first simulation, from Fig. 3, we can see the planar path drown by the vehicle guide points $A_{2}$ and $A_{1}$ of the overtaking and overtaken vehicle, respectively.

TABLE I

COORDINATES OF THE REFERENCE FRAMES $\mathrm{R}_{\mathrm{I}} \mathrm{X}_{\mathrm{RI}} \mathrm{Y}_{\mathrm{RI}},(\mathrm{I}=1,2,3)$, IN OVERTAKEN VEHICLE FRAME $\mathrm{A}_{1} \mathrm{X}_{1} \mathrm{Y}_{1}$

\begin{tabular}{lll}
\hline \hline \multirow{2}{*}{ Phase } & \multicolumn{1}{c}{ Reference Frame } & \multicolumn{1}{c}{ Coordinates } \\
\hline 1 & $\mathrm{R}_{1} \mathrm{x}_{\mathrm{R} 1} \mathrm{y}_{\mathrm{R} 1}$ & $\mathrm{~L}_{1 \mathrm{t}}^{1}=-1 \mathrm{~m} ; \mathrm{L}_{12}^{1}=3 \mathrm{~m}$ \\
2 & $\mathrm{R}_{2} \mathrm{X}_{\mathrm{R} 2} \mathrm{y}_{\mathrm{R} 2}$ & $\mathrm{~L}_{1 \mathrm{t}}^{2}=8 \mathrm{~m} ; \mathrm{L}_{12}^{2}=3 \mathrm{~m}$ \\
3 & $\mathrm{R}_{3} \mathrm{X}_{\mathrm{R} 3} \mathrm{y}_{\mathrm{R} 3}$ & $\mathrm{~L}^{3}{ }_{1 \mathrm{t}}=12 \mathrm{~m} ; \mathrm{L}_{12}^{3}=0 \mathrm{~m}$ \\
\hline \hline
\end{tabular}




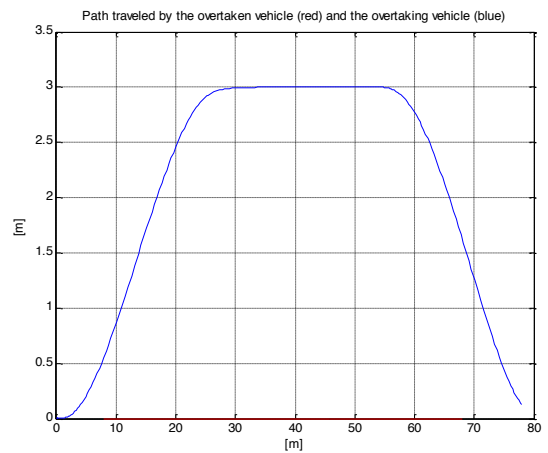

Fig. 3. Planar path drown by the vehicle guide points (the mid-points of the rear vehicle axles): point $A_{2}$ (blue line) and point point $A_{1}$ (red line)

From Fig. 4, we can see the evolution in time of the error coordinates $x_{e}(t), y_{e}(\mathrm{t})$ and $e_{\theta}(t)$.

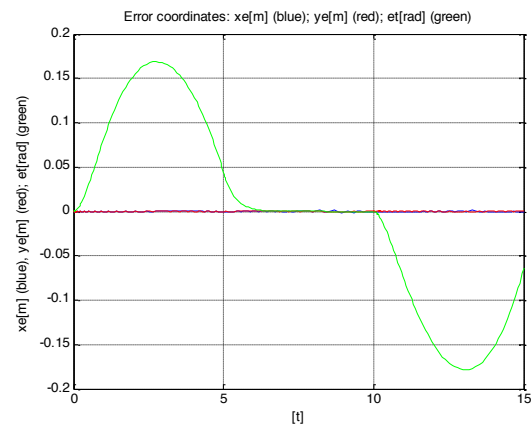

Fig. 4. Time history of the error coordinates $x_{e}(t), y_{e}(\mathrm{t})$ and $e_{\theta}(t)$

As seen from Fig. 4, the relative orientation $e_{\theta}(t)$ between the two vehicles remains bound during the three phases of the overtaking maneuver, which is consistent with the theoretical results in Section IV. The evolution in time of the inter-vehicle distance $A L$ is given in Fig.5.

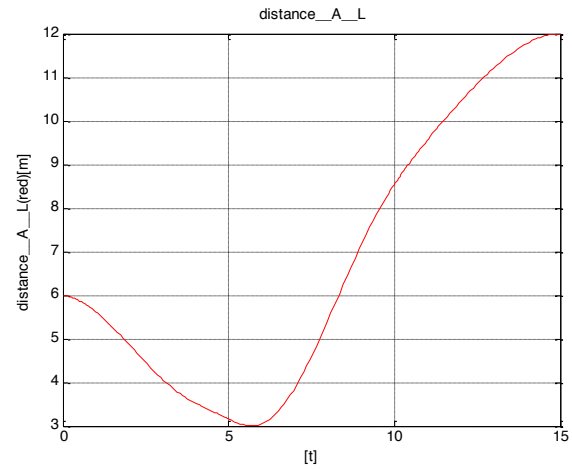

Fig. 5. Time history of the inter-vehicle distance $A L$

An overtaking maneuver involving two $\mathrm{CyCab}$ vehicles has been performed using CIVIC software, as shown in Fig. 6 . The results of the simulation confirm the validity of the proposed controller.

\section{CONCLUSION}

In this paper, a nonlinear adaptive controller for twovehicle three-phase automated overtaking maneuvers has been presented. Reference trajectories for every phase using $3^{\text {rd }}$ order polynomial interpolation method are generated in real-time and consecutively tracked. The only information available for feedback control is the current relative intervehicle position and orientation. The unknown velocity of the overtaken vehicle is estimated using adaptive update law. Simulation results illustrate the performance of the proposed controller. Our future work will include implementation of the proposed controller and experiments with $\mathrm{CyCab}$ vehicles developed at INRIA.

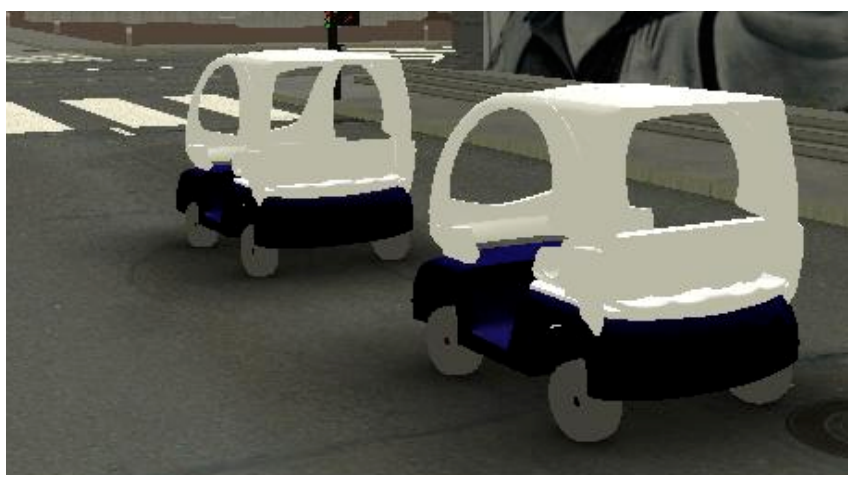

Fig. 6. Overtaking maneuver involving two $\mathrm{CyCab}$ vehicles using CIVIC software

\section{REFERENCES}

[1] V. Cerone, M. Milanese, and D. Regtuto, "Combined automatic lanekeeping and driver's steering trough a 2-DOF control strategy," IEEE Trans. Contr. Syst. Technology, vol. 17, no.1, pp. 135-142, January, 2009.

[2] D. Godbole, R. Sengupta, V. Hagenmeyer, "Distributed hybrid controls for automated vehicle lane changes," in IEEE Conf. Dec. Control, 1998, pp. 2639-2644.

[3] H. Jula, E. Kosmatopoulos, and P. Ioannou, "Collision avoidance analysis for lane changing and merging," California PATH Research Report, UCB-ITS-PRR-99-13, 1999.

[4] J. Naranjo, C. Gonzales, R. Garcia, and T. Pedro, "Lane-change fuzzy control in autonomous vehicles for the overtaking maneuver," IEEE Trans. Intel. Transp. Systems, vol. 9, no. 3, pp. 438-450, September, 2008

[5] J. Baber, J. Kolodko, T. Noel, M. Parent, and L. Vlacic, "Cooperative autonomous driving: Intelligent vehicles sharing city roads," IEEE Robotics \& Automation Magazine, pp. 44-49, March, 2005.

[6] P. Resende and F. Nashashibi, "Real-time dynamic trajectory planning for highly automated driving in highways," in IEEE Conf. Intel. Transp. Systems, 2010, pp. 653-658.

[7] T. Shamir, "Overtaking a slower-moving vehicle by an autonomous vehicle," in ICSC Symp. Eng. Intel. Systems, 2004.

[8] P. Petrov and M. Parent, "Nonlinear adaptive control for autonomous vehicle following," in $16^{\text {th }}$ World Congress Int. Trans. Systems, 2009, paper 3442.

[9] P. Petrov, "A mathematical model for control of an autonomous vehicle convoy," WSEAS Trans. Syst. Control, vol. 3, no. 9, pp. 835848, 2008.

[10] H. Khalil, Nonlinear Systems. Second edition, Prentice-Hall, Inc., 1996. 